\author{
Y. Yilmaz • I. A. Kosal • Y. E. Bakankus
}

\title{
Asymptotic stability for third-order non-homogeneous differential-operator equations
}

Received: 22 April 2017 / Accepted: 27 September 2018 / Published online: 10 October 2018

(C) The Author(s) 2018

\begin{abstract}
In this article, global asymptotic stability of solutions of non-homogeneous differential-operator equations of the third order is studied. It is proved that every solution of the equations decays exponentially under the Routh-Hurwitz criterion for the third order equations.
\end{abstract}

Mathematics Subject Classification $35 \mathrm{~B} 35 \cdot 35 \mathrm{~B} 40 \cdot 35 \mathrm{~B} 45$

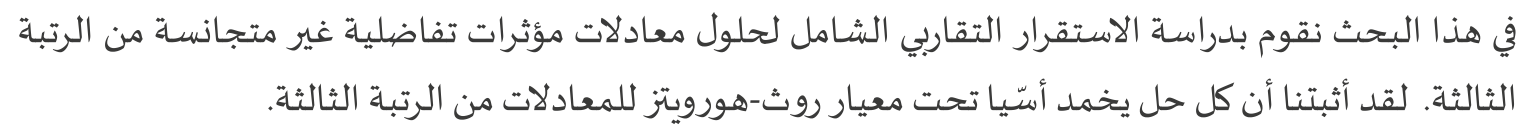

\section{Introduction}

There exist numerous studies on the evolutionary PDEs in the literature. However, works on the differentialoperator equations, especially of the higher order, are rarely encountered [1-3] and [4,7]. Even though there are many works on the stability and instability of solutions to the second-order equations, only a few results are obtained on the stability of solutions to the evolutionary partial differential equations of third order in time.

In [1], the authors have considered an abstract initial value problem to prove the stability of solutions with respect to the initial conditions and the right-hand side function $f(t)$. They obtained global stability results for the non-autonomous second-order differential equations. Kalantarov and Yilmaz have considered a third-order linear differential-operator equation to prove its solutions which tend to zero, backward in time [2]. In [3], Quintanilla and Racke have newly introduced three-phase-lag heat equations in the forms of

$$
\rho c_{\nu} \ddot{T}+\tau_{q} \rho c_{\nu} \dddot{T}=k^{*} \Delta T+\tau_{\nu}^{*} \Delta \dot{T}+k \tau_{T} \Delta \ddot{T}
$$

Y. Yilmaz (凶) · I. A. Kosal · Y. E. Bakankus

Department of Mathematics, Sakarya University, 54187 Adapazari, Turkey

E-mail: yalciny@sakarya.edu.tr

I. A. Kosal

E-mail: isilarda@hotmail.com

Y. E. Bakankus

E-mail: emrebakankus@hotmail.com 
and

$$
\rho c_{\nu} \ddot{T}+\tau_{q} \rho c_{\nu} \dddot{T}+\frac{\tau_{q}^{2}}{2} \rho c_{\nu} \dddot{T}=k^{*} \Delta T+\tau_{\nu}^{*} \Delta \dot{T}+k \tau_{T} \Delta \ddot{T}
$$

For each equation, they give a suitable Lyapunov function, which is a powerful tool to study the qualitative aspects of the solutions of these equations. They have showed that if the stability conditions fail, they can obtain instability solutions for suitable domains. It has also be noted that the two equations are heat and wave type for $\ddot{T}$, respectively.

In [4], the homogeneous differential-operator equation of a third order is investigated. It is shown under some conditions, compatible with the Routh-Hurwitz criterion, that the solution of the equation is asymptotically stable, and that every solution of the equation tends to zero with an exponential rate. For other studies on stability in the third-order problems, one can see [8-12].

In this article, in contrast to the works on homogenous equations mentioned in [2-4], a non-homogeneous differential-operator equation of the third order has been investigated. It has been shown that every solution to the equation is asymptotically stable, provided that a condition on the function on the right-hand side is established.

\section{Preliminaries}

Let $H$ be a real Hilbert space with inner product $(\cdot, \cdot)$ and norm $\|\cdot\|$. We consider in $H$ the following third-order non-homogeneous equation:

$$
u^{\prime \prime \prime}+A u^{\prime \prime}+B u^{\prime}+C u=h(t)
$$

where $A, B$, and $C$ are linear (not necessarily bounded), positively defined, and self-adjoint operators. The unique solvability of the problem can be established by the Faedo-Galerkin method [11]. The domains of these operators $D(A), D(B)$, and $D(C)$ are dense linear subspace of $H$. The symbol "' " stands for differentiation with respect to $t$. Also $\|h(t)\| \rightarrow 0$ as $t \rightarrow \infty$. To show that every solution of (2.1) tends to zero as $t \rightarrow \infty$, we need the following lemma.

Lemma 2.1 Let the operators $A, B$, and $C$ be as above. Suppose that $D(B) \subseteq D(C)$ and there exist positive numbers $\alpha$ and $\gamma_{1}$, such that

$$
\begin{aligned}
& \alpha \gamma_{1}>1, \\
& \alpha\|u\|^{2} \leq(A u, u), \quad \forall u \in D(A)
\end{aligned}
$$

and

$$
(C u, u) \leq \gamma_{1}^{-1}(B u, u), \quad \forall u \in D(B) .
$$

Then, there exists a positive function $\phi(t)$, which is some measure of the solution, satisfying

$$
\phi(u(t)) \geq \sigma\left(\left\|u^{\prime \prime}\right\|^{2}+\left\|A^{1 / 2} u^{\prime}\right\|^{2}+\left\|B^{1 / 2} u^{\prime}\right\|^{2}+\left\|C^{1 / 2} u\right\|^{2}\right) .
$$

Proof Assume that $u=u(t)$ is an arbitrary solution of Eq. (2.1). Taking the inner product in $H$ of (2.1) with $u^{\prime \prime}+\varepsilon u^{\prime}$, where $\varepsilon$ is a positive number specified below, and using the standard inequality to the right-hand side, we obtain

$$
\begin{aligned}
& \frac{1}{2} \frac{d}{d t}\left\|u^{\prime \prime}\right\|^{2}+\left\|A^{1 / 2} u^{\prime \prime}\right\|^{2}+\frac{1}{2} \frac{d}{d t}\left\|B^{1 / 2} u^{\prime}\right\|^{2}+\left(C u, u^{\prime \prime}\right)+\varepsilon\left(u^{\prime \prime \prime}, u^{\prime}\right) \\
& \quad+\varepsilon\left\|B^{1 / 2} u^{\prime}\right\|^{2}+\frac{\varepsilon}{2} \frac{d}{d t}\left\|A^{1 / 2} u^{\prime}\right\|^{2}+\frac{\varepsilon}{2} \frac{d}{d t}\left\|C^{1 / 2} u\right\|^{2}-\varepsilon_{1}\left\|A^{1 / 2} u^{\prime \prime}\right\|^{2} \\
& \quad-\varepsilon^{2} \varepsilon_{2}\left\|B^{1 / 2} u^{\prime}\right\|^{2} \leq \frac{1}{4 \varepsilon_{1}}\left\|A^{-1 / 2} h\right\|^{2}+\frac{1}{4 \varepsilon_{2}}\left\|B^{-1 / 2} h\right\|^{2},
\end{aligned}
$$


where $\varepsilon_{1}$ and $\varepsilon_{2}$ are positive numbers which are specified below. It follows that

$$
\begin{aligned}
& \frac{d}{\mathrm{~d} t}\left[\frac{1}{2}\left\|u^{\prime \prime}\right\|^{2}+\frac{1}{2}\left\|B^{1 / 2} u^{\prime}\right\|^{2}+\frac{\varepsilon}{2}\left\|A^{1 / 2} u^{\prime}\right\|^{2}+\frac{\varepsilon}{2}\left\|C^{1 / 2} u\right\|^{2}+\varepsilon\left(u^{\prime \prime}, u^{\prime}\right)\right. \\
& \left.+\left(C^{1 / 2} u, C^{1 / 2} u^{\prime}\right)\right]+\left\|A^{1 / 2} u^{\prime \prime}\right\|^{2}+\varepsilon\left\|B^{1 / 2} u^{\prime}\right\|^{2}-\varepsilon\left\|u^{\prime \prime}\right\|^{2}-\left\|C^{1 / 2} u^{\prime}\right\|^{2} \\
& -\varepsilon_{1}\left\|A^{1 / 2} u^{\prime \prime}\right\|^{2}-\varepsilon^{2} \varepsilon_{2}\left\|B^{1 / 2} u^{\prime}\right\|^{2} \leq \frac{1}{4 \varepsilon_{1}}\left\|A^{-1 / 2} h\right\|^{2}+\frac{1}{4 \varepsilon_{2}}\left\|B^{-1 / 2} h\right\|^{2} .
\end{aligned}
$$

If we denote

$$
\begin{aligned}
\phi(u(t)):= & \frac{1}{2}\left\|u^{\prime \prime}\right\|^{2}+\frac{1}{2}\left\|B^{1 / 2} u^{\prime}\right\|^{2}+\frac{\varepsilon}{2}\left\|A^{1 / 2} u^{\prime}\right\|^{2} \\
& +\frac{\varepsilon}{2}\left\|C^{1 / 2} u\right\|^{2}+\varepsilon\left(u^{\prime \prime}, u^{\prime}\right)+\left(C^{1 / 2} u, C^{1 / 2} u^{\prime}\right),
\end{aligned}
$$

then the last inequality becomes

$$
\begin{aligned}
& \frac{d}{\mathrm{~d} t} \phi(u(t))+\left\|A^{1 / 2} u^{\prime \prime}\right\|^{2}+\varepsilon\left\|B^{1 / 2} u^{\prime}\right\|^{2}-\varepsilon\left\|u^{\prime \prime}\right\|^{2}-\left\|C^{1 / 2} u^{\prime}\right\|^{2} \\
& \quad-\varepsilon_{1}\left\|A^{1 / 2} u^{\prime \prime}\right\|^{2}-\varepsilon^{2} \varepsilon_{2}\left\|B^{1 / 2} u^{\prime}\right\|^{2} \leq \frac{1}{4 \varepsilon_{1}}\left\|A^{-1 / 2} h\right\|^{2}+\frac{1}{4 \varepsilon_{2}}\left\|B^{-1 / 2} h\right\|^{2} .
\end{aligned}
$$

Using the Cauchy inequality, together with conditions (2.3) and (2.4), we get

$$
\begin{aligned}
\varepsilon\left|\left(u^{\prime \prime}, u^{\prime}\right)\right| & \leq \frac{1}{2\left(1+\varepsilon_{3}\right)}\left\|u^{\prime \prime}\right\|^{2}+\frac{\varepsilon^{2}\left(1+\varepsilon_{3}\right)}{2 \alpha}\left\|A^{1 / 2} u^{\prime}\right\|^{2} \\
\left|\left(C^{1 / 2} u, C^{1 / 2} u^{\prime}\right)\right| & \leq \gamma_{1}^{-1 / 2}\left\|C^{1 / 2} u\right\|\left\|B^{1 / 2} u^{\prime}\right\| \\
& \leq \frac{1-\varepsilon_{4}}{2}\left\|B^{1 / 2} u^{\prime}\right\|^{2}+\frac{1}{2 \gamma_{1}\left(1-\varepsilon_{4}\right)}\left\|C^{1 / 2} u\right\|^{2},
\end{aligned}
$$

where $\varepsilon_{3}$ and $\varepsilon_{4}$ are constants to be chosen below. Hence, due to (2.8) and (2.9), we find the following estimate for $\phi(u(t))$ :

$$
\begin{aligned}
\phi(u(t)) \geq & \frac{1}{2} \frac{\varepsilon_{3}}{1+\varepsilon_{3}}\left\|u^{\prime \prime}\right\|^{2}+\frac{1}{2}\left(\varepsilon-\frac{\varepsilon^{2}\left(1+\varepsilon_{3}\right)}{\alpha}\right)\left\|A^{1 / 2} u^{\prime}\right\|^{2} \\
& +\frac{\varepsilon_{4}}{2}\left\|B^{1 / 2} u^{\prime}\right\|^{2}+\frac{1}{2}\left(\varepsilon-\frac{1}{\gamma_{1}\left(1-\varepsilon_{4}\right)}\right)\left\|C^{1 / 2} u\right\|^{2} .
\end{aligned}
$$

From condition (2.2), it is clear that there is a positive number $\alpha^{\prime}<\alpha$, such that

$$
\alpha^{\prime} \gamma_{1}>1
$$

We can also choose $\varepsilon_{3}$ and $\varepsilon_{4}$, such that

$$
d_{0} \equiv \frac{1}{2}\left[\alpha^{\prime}-\frac{\left(\alpha^{\prime}\right)^{2}\left(1+\varepsilon_{3}\right)}{\alpha}\right]
$$

and

$$
d_{1} \equiv \frac{1}{2 \gamma_{1}}\left[\alpha^{\prime} \gamma_{1}-\frac{1}{1-\varepsilon_{4}}\right]
$$

are positive. Taking $\varepsilon=\alpha^{\prime}$ in (2.10), we obtain

$$
\phi(u(t)) \geq \frac{1}{2} \frac{\varepsilon_{3}}{1+\varepsilon_{3}}\left\|u^{\prime \prime}\right\|^{2}+\frac{\varepsilon_{4}}{2}\left\|B^{1 / 2} u^{\prime}\right\|^{2}+d_{0}\left\|A^{1 / 2} u^{\prime}\right\|^{2}+d_{1}\left\|C^{1 / 2} u\right\|^{2},
$$

and we see that

$$
\phi(u(t)) \geq 0
$$


For some specific works on the stability for the third-order problem, one can see [4] and [12].

Theorem 2.2 Suppose that all conditions of lemma are satisfied. Assume also that $D(B)=D(C) \subseteq D(A)$, and there exist positive numbers $\beta, \gamma_{2}$, and $\alpha_{1}$, such that

$$
\begin{aligned}
& \beta(A u, u) \leq(B u, u), \quad \forall u \in D(B) \\
& \gamma_{2}(B u, u) \leq(C u, u), \quad \forall u \in D(B)
\end{aligned}
$$

and

$$
(A u, u) \leq \alpha_{1}^{-1}\|u\|^{2}, \quad \forall u \in D(A),
$$

and then, every solution of Eq. (2.1) is asymptotically stable in the sense of the norm:

$$
\left\|u^{\prime \prime}\right\|^{2}+\left\|B^{-1 / 2} u^{\prime}\right\|^{2}+\left\|C^{-1 / 2} u\right\|^{2} .
$$

Moreover, every solution of the Cauchy problem for Eq. (2.1) tends to zero as $t \rightarrow \infty$.

Proof Let us take the scalar product in $H$ of (2.1) with $\eta u$. Using the standard inequality, we obtain

$$
\begin{aligned}
& \frac{d}{\mathrm{~d} t}\left[\eta\left(u^{\prime \prime}, u\right)-\frac{\eta}{2}\left\|u^{\prime}\right\|^{2}+\eta\left(A^{1 / 2} u^{\prime}, A^{1 / 2} u\right)+\frac{\eta}{2}\left\|B^{1 / 2} u\right\|^{2}\right] \\
& \quad+\eta\left\|C^{1 / 2} u\right\|^{2}-\eta\left\|A^{1 / 2} u^{\prime}\right\|^{2}-\eta \varepsilon_{5}\left\|C^{1 / 2} u\right\|^{2} \leq \frac{\eta}{4 \varepsilon_{5}}\left\|C^{-1 / 2} h\right\|^{2},
\end{aligned}
$$

where $\eta$ is a positive parameter to be chosen later. Considering that (2.18) and (2.8) together give

$$
\begin{aligned}
\frac{d}{\mathrm{~d} t} & {\left[\phi(u)+\eta\left(u^{\prime \prime}, u\right)-\frac{\eta}{2}\left\|u^{\prime}\right\|^{2}+\eta\left(A^{1 / 2} u^{\prime}, A^{1 / 2} u\right)+\frac{\eta}{2}\left\|B^{1 / 2} u\right\|^{2}\right] } \\
& +\left.\left\|A^{1 / 2} u^{\prime \prime}\right\|\right|^{2}+\alpha^{\prime}\left\|B^{1 / 2} u^{\prime}\right\|^{2}-\alpha^{\prime}\left\|u^{\prime \prime}\right\|^{2}-\left\|C^{1 / 2} u^{\prime}\right\|^{2}-\varepsilon_{1}\left\|A^{1 / 2} u^{\prime \prime}\right\|^{2} \\
& -\left(\alpha^{\prime}\right)^{2} \varepsilon_{2}\left\|B^{1 / 2} u^{\prime}\right\|^{2}+\eta\left\|C^{1 / 2} u\right\|^{2}-\eta\left\|A^{1 / 2} u^{\prime}\right\|^{2}-\eta \varepsilon_{5}\left\|C^{1 / 2} u\right\|^{2} \\
\leq & \frac{1}{4 \varepsilon_{1}}\left\|A^{-1 / 2} h\right\|^{2}+\frac{1}{4 \varepsilon_{2}}\left\|B^{-1 / 2} h\right\|^{2}+\eta \frac{1}{4 \varepsilon_{5}}\left\|C^{-1 / 2} h\right\|^{2} .
\end{aligned}
$$

Let us denote $\psi(u(t))$ as

$$
\psi(u(t)):=\phi(u)+\eta\left(u^{\prime \prime}, u\right)-\frac{\eta}{2}\left\|u^{\prime}\right\|^{2}+\eta\left(A^{1 / 2} u^{\prime}, A^{1 / 2} u\right)+\frac{\eta}{2}\left\|B^{1 / 2} u\right\|^{2} .
$$

Rewriting (2.20) by considering (2.12) gives

$$
\begin{gathered}
\psi(u(t)) \geq \frac{1}{2} \frac{\varepsilon_{3}}{1+\varepsilon_{3}}\left\|u^{\prime \prime}\right\|^{2}+\frac{\varepsilon_{4}}{2}\left\|B^{1 / 2} u^{\prime}\right\|^{2}+d_{0}\left\|A^{1 / 2} u^{\prime}\right\|^{2}+d_{1}\left\|C^{1 / 2} u\right\|^{2} \\
+\eta\left(u^{\prime \prime}, u\right)-\frac{\eta}{2}\left\|u^{\prime}\right\|^{2}+\eta\left(A^{1 / 2} u^{\prime}, A^{1 / 2} u\right)+\frac{\eta}{2}\left\|B^{1 / 2} u\right\|^{2} .
\end{gathered}
$$

Using (2.14), (2.15), and the Cauchy inequality, one obtains

$$
\begin{aligned}
\psi(u(t)) \geq & \frac{1}{2}\left(\frac{\varepsilon_{3}}{1+\varepsilon_{3}}-\frac{\eta}{a}\right)\left\|u^{\prime \prime}\right\|^{2}+\frac{\eta}{2}\left(1-\frac{\alpha}{\alpha \beta}-\frac{b}{\beta}\right)\left\|B^{1 / 2} u\right\|^{2} \\
& +d_{1}\left\|C^{1 / 2} u\right\|^{2}+\frac{\varepsilon_{4}}{2}\left\|B^{1 / 2} u^{\prime}\right\|^{2}+\left(d_{0}-\frac{\eta}{2 \alpha}-\frac{\eta}{2 b}\right)\left\|A^{1 / 2} u^{\prime}\right\|^{2},
\end{aligned}
$$

where

$$
\eta\left|\left(u^{\prime \prime}, u\right)\right| \leq \frac{\eta}{2 a}\left\|u^{\prime \prime}\right\|^{2}+\frac{\eta a}{2 \alpha \beta}\left\|B^{1 / 2} u\right\|^{2}
$$

and

$$
\eta\left|\left(A^{1 / 2} u^{\prime}, A^{1 / 2} u\right)\right| \leq \frac{\eta}{2 b}\left\|A^{1 / 2} u^{\prime}\right\|^{2}+\frac{b \eta}{2 \beta}\left\|B^{1 / 2} u\right\|^{2} .
$$


If we first choose $\eta=\min \left(\frac{a \varepsilon_{3}}{1+\varepsilon_{3}}, \frac{2 \alpha b d_{0}}{\alpha+b}\right)$, then $a=\frac{\alpha \beta}{2}$ and $b=\frac{\beta}{2}$ give us

$$
\psi(u(t)) \geq v_{1}\left(\left\|u^{\prime \prime}\right\|^{2}+\left\|B^{1 / 2} u^{\prime}\right\|^{2}+\left\|C^{1 / 2} u\right\|^{2}\right),
$$

where $v_{1}=\min \left\{\frac{1}{2}\left(\frac{\varepsilon_{3}}{1+\varepsilon_{3}}-\frac{\eta_{0}}{a}\right), d_{1}, \frac{\varepsilon_{4}}{2}, d_{0}-\frac{\eta(\alpha+b)}{2 \alpha b}\right\}$ is a positive parameter depending on $\alpha, \beta$, $\gamma_{1}, \gamma_{2}$. In addition, it could be easily verified that, with the parameter $\nu_{2}=v_{2}\left(\alpha, \beta, \gamma_{1}, \gamma_{2}\right)$,

$$
\psi(u(t)) \leq v_{2}\left(\left\|u^{\prime \prime}\right\|^{2}+\left\|B^{1 / 2} u^{\prime}\right\|^{2}+\left\|C^{1 / 2} u\right\|^{2}\right)
$$

for each solution $u(t)$ of Eq. (2.1). By using (2.3), (2.4), (2.14), (2.15), and (2.16), we can obtain the following inequality from (2.19), (2.20), and (2.22):

$$
\begin{aligned}
\frac{d}{\mathrm{~d} t} \psi(u(t))+\delta \psi(u(t)) \leq & -\left(\alpha-\alpha^{\prime}-\varepsilon_{1} \alpha_{1}^{-1}-\delta v_{2}\right)\left\|u^{\prime \prime}\right\|^{2} \\
& -\left(\eta-\eta \varepsilon_{5}-\delta v_{2}\right)\left\|C^{1 / 2} u\right\|^{2} \\
& -\left(\alpha^{\prime}-\gamma_{1}^{-1}-\left(\alpha^{\prime}\right)^{2} \varepsilon_{2}-\eta \beta^{-1}-\delta v_{2}\right)\left\|B^{1 / 2} u^{\prime}\right\|^{2} \\
& +\frac{1}{4 \varepsilon_{1}}\left\|A^{-1 / 2} h\right\|^{2}+\frac{1}{4 \varepsilon_{2}}\left\|B^{-1 / 2} h\right\|^{2}+\eta \frac{1}{4 \varepsilon_{5}}\left\|C^{-1 / 2} h\right\|^{2} .
\end{aligned}
$$

Since $\alpha$ and $\alpha^{\prime}$ satisfy (2.2) and (2.11), respectively, $\delta$ and $\eta$ can be chosen so small that

$$
\frac{d}{\mathrm{~d} t} \psi(u(t))+\delta \psi(u(t)) \leq \frac{1}{4 \varepsilon_{1}}\left\|A^{-1 / 2} h\right\|^{2}+\frac{1}{4 \varepsilon_{2}}\left\|B^{-1 / 2} h\right\|^{2}+\eta \frac{1}{4 \varepsilon_{5}}\left\|C^{-1 / 2} h\right\|^{2} .
$$

Let us denote the right-hand side of (2.23) by $\varphi(t)$. Multiplying both sides of (2.23) by $e^{\delta t}$ and integrating on $(0, t)$, we obtain

$$
\psi(u(t)) \leq e^{-\delta t}\left(\psi(u(0))+\int_{0}^{t} e^{\delta \tau} \varphi(\tau) d \tau\right) .
$$

Since $\|h(t)\| \rightarrow 0$ as $t \rightarrow \infty$, it follows that $\psi(u(t)) \rightarrow 0$. Some measure of Eq. (2.1) tends to zero. Moreover, every solution of the Cauchy problem tends to zero with an exponential rate. Hence, the proof is completed. $\square$

\section{Applications}

Application 3.1 One of the models describing acoustic waves in a non-uniform compressible relaxing medium whose density in the unperturbed state depends only on $x$ is given by the following:

$$
\tau(x) \frac{\partial}{\partial t}\left(\frac{\partial^{2} p}{\partial t^{2}}-C_{\infty}^{2}(x) \Delta p\right)+\frac{\partial^{2} p}{\partial t^{2}}-C_{0}^{2}(x) \triangle p=0 .
$$

For more detailed physical interpretation, existence, and uniqueness of the solutions, we refer to [10]. It can be stated that (3.1) differs from the equation for acoustic waves in uniform medium only by the fact that the coefficients are not constant. Taking into account one-dimensional case of (3.1) in $x$, introducing $x=x /\left[C_{\infty}(0) T_{i}\right]$ and $t=t / T_{i}$ as dimensionless variables, where $T_{i}$ is the characteristic period of internal interactions, and setting $\gamma(x)=T_{i} / \tau(x), \kappa(x)=\left[C_{\infty}(x) / C_{\infty}(0)\right]^{2}, \alpha(x)=\left[C_{0}(x) / C_{\infty}(0)\right]^{2}$ and $u=p / p(0) C_{\infty}^{2}(0)$, we lead to the following non-homogeneous initial-boundary value problem:

$$
\begin{aligned}
& \frac{\partial^{3} u}{\partial t^{3}}+\gamma(x) \frac{\partial^{2} u}{\partial t^{2}}-\kappa(x) \frac{\partial^{3} u}{\partial x^{2} \partial t}-\alpha(x) \gamma(x) \frac{\partial^{2} u}{\partial x^{2}}=f(x, t) \\
& (x, t) \in \Omega_{T}=\{x>0,0<t<T<\infty\} \\
& u(x, 0)=u^{0}(x), u_{t}(x, 0)=u^{I}(x), u_{t t}(x, 0)=u^{I I}(x), \quad x>0 \\
& u(0, t)=\phi(t), \quad t>0 .
\end{aligned}
$$


Taking $A=\gamma(x) I, B=-\kappa(x) \frac{\partial^{2}}{\partial x^{2}}$ and $C=-\alpha(x) \kappa(x) \frac{\partial^{2}}{\partial x^{2}}$, Eq. (3.2) can be rewritten in the form of (2.1) as follows:

$$
u_{t t t}+A u_{t t}+B u_{t}+C u=f(x, t) .
$$

Thus, Theorem 2.2 allows us the possibility to confirm that, under the required conditions on the function $f(x, t)$, every solution of (3.4) satisfying the conditions in (3.3) tends to zero with an exponential rate.

Application 3.2 Any physical process including the photon interactions in metal films and photon scattering in dielectric media occurs in a finite time. These microscopic interactions are viewed as impeding sources causing a delayed response on the macroscopic scale. In [9], the heat flux is eliminated using the Taylor series expansion with respect to $t$ by considering the one-dimensional equation obtained in [9], which shows that the heat flux and the temperature gradient occur in a sequence of time, and by employing the conservation of energy, the following equation is derived

$$
\frac{\partial^{2} T}{\partial x^{2}}+\tau_{T} \frac{\partial^{3} T}{\partial x^{2} \partial t}+\frac{1}{K}\left(S+\tau_{q} \frac{\partial S}{\partial t}+\frac{\tau_{q}^{2}}{2} \frac{\partial^{2} S}{\partial t^{2}}\right)=\frac{1}{\alpha} \frac{\partial T}{\partial t}+\frac{\tau_{q}}{\alpha} \frac{\partial^{2} T}{\partial t^{2}}+\frac{\tau_{q}^{2}}{2 \alpha} \frac{\partial^{3} T}{\partial t^{3}},
$$

which predicts wave-like thermal signals propagating with the finite speed. Equation (3.5) forms a macroscopic dual-phase lag model. A similar microscopic hyperbolic two-step model, which is obtained from three coupled equations arrived in [6], can be found in [9].

For simplicity, we introduce $t=t / \tau_{q}$ and $x=x / \sqrt{\alpha \tau_{q}}$ as dimensionless variables. Setting $T=\frac{T-T_{0}}{T_{\omega}-T_{0}}$, where $T_{\omega}$ is the suddenly raised temperature at the front surface at $x=0$ and $T_{0}$ is the initial temperature of the medium as $t=0$, and $S=\frac{\alpha \tau_{q}}{T_{\omega}-T_{0}} S$, Eq. (3.5) can be rewritten as follows:

$$
\frac{\partial^{3} T}{\partial t^{3}}+2 \frac{\partial^{2} T}{\partial t^{2}}+2\left(I-R \frac{\partial^{2}}{\partial x^{2}}\right) \frac{\partial T}{\partial t}-2 \frac{\partial^{2}}{\partial x^{2}} T=\frac{1}{K}\left(\frac{\partial^{2} S}{\partial t^{2}}+2 \frac{\partial S}{\partial t}+2 S\right),
$$

where $I$ is the unit operator and $R=\tau_{T} / \tau_{q}$. Taking $A=2 I, B=2\left(I-R \frac{\partial^{2}}{\partial x^{2}}\right), C=-2 \frac{\partial^{2}}{\partial x^{2}}$ and $f(x, t)=\frac{1}{K}\left(\frac{\partial^{2} S}{\partial t^{2}}+2 \frac{\partial S}{\partial t}+2 S\right)$, Eq. (3.6) can be written in the form in (2.1) as follows:

$$
T_{t t t}+A T_{t t}+B T_{t}+C T=f(x, t) .
$$

By Theorem 2.2, every solution of (3.7) tends to zero with an exponential rate if the required conditions on $f(x, t)$ and the conditions in (3.3) are satisfied.

Open Access This article is distributed under the terms of the Creative Commons Attribution 4.0 International License (http:// creativecommons.org/licenses/by/4.0/), which permits unrestricted use, distribution, and reproduction in any medium, provided you give appropriate credit to the original author(s) and the source, provide a link to the Creative Commons license, and indicate if changes were made.

\section{References}

1. Iovanovich, B.S.; Lemeshevski, S.V.; Matus, P.P.: Global stability of second-order operator-differential equations and threelayer operator-difference schemes. Dokl. Nats. Akad. Nauk Belarusi 46(4), 30-34 (2002). 124

2. Kalantarov, V.K.; Yilmaz, Y.: Decay and growth estimates for solutions of second-order and third-order differential-operator equations. Nonlinear Anal. 89, 1-7 (2013)

3. Quintanilla, R.; Racke, R.: A note on stability in three-phase-lag heat con- duction. Int. J. Heat and Mass Transf. 51, 24-29 (2008)

4. Kalantarov, V.K.; Tiryaki, A.: On the stability results for third-order differential-operator equations. Turk. J. Math. 21, 179-186 (1997) 
5. Ladyzhenskaya, O.A.: On non-stationary operational equations and their applications to linear problems of mathematical physics. Mat. Sb. 45, 123-149 (1985)

6. Temam, R.: Infinite-dimensional dynamical systems in mechanics and physics. Applied Mathematical Sciences, vol. 68. Springer, New York (1997)

7. Tzou, D.Y.: The generalized lagging response in small-scale and high-rate heating. Int. J. Heat Mass Transf. 38(17), 3231$3240(1995)$

8. Varlamov, V.V.; Nesterov, A.V.: Asymptotic representation of the solution of the problem of the propagation of acoustic waves in a non-uniform compressible relaxing medium. USSR Comput. Math. Math. Phys. 30(3), 47-55 (1990)

9. Marchand, R.; McDevitt, T.; Triggiani, R.: An abstract semigroup approach to the third-order Moore-Gibson-Thompson partial differential equation arising in high-intensity ultrasound: structural decomposition, spectral analysis, exponential stability. Math. Methods Appl. Sci. 35(15), 1896-1929 (2012)

10. Remili, M.; Beldjerd, D.: Stability and ultimate boundedness of solutions of some third order differential equations with delay. J Assoc. Arab Univ. Basic Appl. Sci. 23, 90-95 (2017)

11. Graef, J.R.; Tunç, C.: Global asymptotic stability and boundedness of certain multi-delay functional differential equations of third order. Math. Methods Appl. Sci. 38(17), 3747-3752 (2015)

12. Tunç, C.: On the qualitative behaviors of nonlinear functional differential systems of third order. Advances in nonlinear analysis via the concept of measure of noncompactness, 421-439. Springer, Singapore (2017)

Publisher's Note Springer Nature remains neutral with regard to jurisdictional claims in published maps and institutional affiliations. 